\title{
Liderazgo resonante. Un análisis a través de grupos de discusión.
}

\author{
Beatriz PeÑa AcuÑa \\ UCAM \\ bpena@ucam.edu \\ María Concepción PARra-Meroño \\ UCAM \\ mcparra@ucam.edu \\ Miguel Ángel Beltrán Bueno \\ UCAM \\ mabeltran@ucam.edu
}

\section{Resumen:}

El presente artículo pretende adentrarse en el análisis cualitativo- a través de los resultados de varios grupos de discusión- con el fin de descubrir de qué modo este tipo de liderazgo es percibido por jóvenes que han alternado los estudios con un trabajo parcial y poseen cierta experiencia profesional y de trabajo en equipo. Este tipo de liderazgo surge en un momento sociocultural donde la inteligencia emocional se ha hecho ya un lugar común en las escuelas, mundo empresarial y esfera personal, en una ocasión donde este tipo de inteligencia se promueve, es estima y se descubre cada vez más como necesario.

Palabras clave: inteligencia emociona; liderazgo resonante; empresa; investigación; desarrollo personal.

\section{Resonant Leadership. An analysis through discussion groups.}

\begin{abstract}
:
This article aims to delve into the qualitative analysis, through the results of several focus groups, in order to discover how such leadership is perceived by young people who have alternated studies with part-time work and have some professional experience and teamwork. This type of leadership comes at a cultural moment where emotional intelligence has become a commonplace in schools, business and personal, in an occasion where this type of intelligence is promoted, estimated and found increasingly as necessary.
\end{abstract}

Key Words: emotional intelligence; resonant leadership; business; research; personal development.

\section{Referencia normalizada:}

Peña Acuña, B., Parra Meroño, M.C. y Beltrán Bueno, M.A. (2014): Liderazgo resonante. Un análisis de grupos de discusión. Historia y Comunicación Social. Vol. 19. Núm. Especial Enero. Págs. 143-151.

Sumario: 1. Introducción. 2. Estado de la cuestión 3. Marco conceptual. 4. Resultados. 5. Conclusiones. 6. Bibliografía. 


\section{Introducción}

En este artículo pretendemos averiguar hasta qué punto el liderazgo resonante es estimado como un liderazgo plausible por parte de empleados españoles. En otra publicación anterior (2013) titulada "Liderazgo resonante en el mundo empresarial" ya expusimos una introducción acerca del liderazgo resonante y de los diversos aspectos que lo influyen desde la inteligencia emocional y la social, que a nuestro juicio debe poseer el directivo empresarial y en los que resulta impactado el empleado. Además se exponían varios casos de empresas en los que el liderazgo resonante había mejorado el ambiente de trabajo y el rendimiento de los empleados.

Recordamos solo brevemente que el concepto de liderazgo resonante fue formulado por Daniel Goleman, R. Boyatzis y A. McKee en 2005 como una de las derivaciones de la inteligencia emocional publicitado en 1995 por el mismo Daniel Goleman, pero esta vez aplicado al mundo empresarial. Estos autores explican de qué manera el sistema límbico -también llamado cerebro emocional- influye en nuestra vida en mayor medida que la corteza cerebral -parte racional del cerebro- y por qué el estado de ánimo de los líderes tiene un efecto tan profundo en las personas a las que lideran.

Dichos autores afirman que la tarea fundamental del líder es despertar los sentimientos positivos de sus subordinados y ello ocurre cuando un líder produce resonancia -sintoniza mejor con los demás y mantiene relaciones transparentes-. Este tipo de líder está capacitado para proporcionar a los demás pautas para interpretar y reaccionar emocionalmente ante determinadas situaciones. En este sentido es el principal «gestor de significado» de un grupo. Por ejemplo, en contextos de crisis o turbulencia todas las miradas convergen en el líder en busca de orientación y de algún modo desempeña un papel fundamental en el clima emocional colectivo ya que explícita o implícitamente determina la norma emocional del grupo. Algunos de los signos más ventajosos del líder resonante consisten en el optimismo y el entusiasmo que exhiben sus subordinados.

\section{Estado de la cuestión}

El liderazgo resonante es un tipo de liderazgo que goza de repercusión especial en el mundo empresarial de los EE.UU donde fue enunciado y difundido en un principio, y cuya influencia recibimos como legado cultural en Europa, y en España en concreto. Todavía son recientes y muchas las publicaciones que se producen en inglés por parte de Goleman, McKee, Caruso, Salovey (consultar bibliografía) y la traducción al castellano en las que se destacan las ventajas de este tipo de liderazgo y la repercusión del despliegue la de la inteligencia emocional.

La investigación que hemos llevado a cabo pretende aportar un poco de luz a qué estima recibe este tipo de liderazgo en los empleados españoles y de algún modo señala que tipo de aceptación e implantación puede tener entre las generaciones de 
jóvenes. El estudio se ha llevado a cabo en una muestra de 40 alumnos de último curso de carrera de Administración y Dirección de Empresas de 22 años de media, que habían tenido ya experiencia profesional compatibilizada con sus estudios.

La metodología escogida para la investigación fue cualitativa. El estudio consistió en la realización de cuatro grupos de discusión (también llamados focus group). Estos grupos estaban compuestos por diez personas en los que discutieron y expusieron las ventajas e inconvenientes de este liderazgo después de haber estudiado durante un semestre acerca de las diversas teorías de liderazgo en la asignatura de "Dirección de Comunicación y Habilidades Directivas" que se imparte en cuarto curso del Grado en Administración y Dirección de Empresas. Además, debían valorar la teoría del liderazgo resonante y ponerla en relación a su propia experiencia profesional de modo vivencial.

En esta pesquisa se les pedía que dieran su opinión acerca de las ventajas e inconvenientes del liderazgo resonante respecto a la inteligencia emocional y la inteligencia social. Cada grupo expuso en clase los principales argumentos mediante un portavoz que reunió las principales ideas y además apuntó todas estas ideas a medida que eran expuestas por los integrantes del grupo de discusión.

\section{Marco conceptual}

Como se ha indicado anteriormente, el concepto de liderazgo resonante se deriva del de Inteligencia Emocional. Los estudios de Peter Alovey y John Mayer sobre inteligencia emocional, publicados en 1990, permiten identificar los componentes del coeficiente emocional, a saber, la autoconfianza, el autocontrol, persistencia, la empatía y el dominio de las relaciones. Los tres primeros indicadores, se refieren a la gestión de uno mismo y se relacionan con la motivación al logro; los dos últimos son competencias relativas a la afiliación y el poder social, siendo las más difíciles de desarrollar. En concreto, la autoconfianza consiste en conocer las propias emociones. El autocontrol está referido a la capacidad de cambiar o frenar emociones. La persistencia es la capacidad de estimularse ante situaciones adversas.

Además, Goleman, gran difusor de la inteligencia emocional y de la inteligencia resonante enuncia que, dentro de las habilidades sociales lo más importante es la influencia sobre los demás (en la difusión del liderazgo resonante, 2003), comunicarse de una manera eficaz, dirigir de una manera humilde grupos de trabajo y resolver conflictos negociándolos y cooperando.

Más adelante, Goleman ha vuelto a hablar de otro tipo de inteligencia: la inteligencia social (2006), entendida como la capacidad humana de relacionarse, integrada por la sensibilidad social que incluye la empatía o comprensión de los sentimientos por otros y la capacidad de relación, que facilita el desarrollo de la sensibilidad social. 


\section{Resultados}

En este epígrafe se detallan los resultados derivados de los grupos de discusión realizados sobre la temática de la investigación.

En cuanto a las ventajas del liderazgo resonante, el primer grupo consideraba una ventaja el hecho de que este tipo de líder en un grupo ayudaba en el desempeño del grupo. Por otro lado, el hecho de que el líder fuera optimista y entusiasta motivaba al grupo a serlo también. Consideraban que este tipo de apoyo para los subordinados era un apoyo central. Además se creaba confianza entre el líder y los subordinados, y si cabe, armonía entre los trabajadores. Otra de las ventajas es que el líder es capaz de controlar sus emociones. Como conclusión, el líder resonante debe tener claro la responsabilidad que es tener este papel ante un grupo en específico, ya que tiene un gran peso sobre él. Debe saber motivar y llevar a un progreso de manera positiva, para cumplir, en este caso, con los objetivos de la empresa.

El segundo grupo considera que el líder resonante es aquel que genera emociones positivas en sus subordinados a través del cultivo de tres factores, a saber, conciencia, esperanza y compasión. Entre las ventajas que desarrolla la inteligencia emocional enumeran: que la comunicación mejora, que el trabajador se siente más persona y con más calidad de vida, que se aumenta la motivación, que las relaciones personales mejoran, que las personas se implican más en el trabajo y son más responsables y autónomas, que se produce una mejora en el clima laboral, que el poder y el liderazgo se ven reforzados, que aumenta la eficiencia de las personas y los equipos, que los procesos de cambio y de mejora continua se agilizan, que se mejoran las relaciones con los clientes y como consecuencia mejora la rentabilidad de la empresa. Además, este grupo de discusión estima conveniente desplegar ciertas actitudes para obtener una buena inteligencia social como la empatía, el liderazgo, la competencia verbal, la inteligencia emocional, la asertividad, saber escuchar y prestar atención, ser atinado para analizar el lenguaje corporal de los demás, ser bueno psicoanalizando y leer entre líneas cuando los demás hablan, gestionar bien el contacto físico e interpretar correctamente las situaciones sociales que hubiere alrededor.

El tercer grupo destacaba también como ventaja de este tipo de liderazgo la capacidad del líder de despertar sentimientos positivos de los subordinados y además que desplegara capacidad de motivar. Consideran también que el líder proporciona pautas para interpretar y reaccionar emocionalmente ante determinadas situaciones. Esto permite dotar a los empleados de inteligencia emocional también, por ejemplo, en situaciones de crisis económica, un buen líder permite que su equipo caiga en una situación de desánimo, por el contrario, motiva e incentiva al equipo para que aporten ideas, iniciativas y propuestas para salir de esa situación. Además, el líder determina la norma emocional del grupo. Respecto a la inteligencia social, el líder resonante, aplicando bien sus tareas, consigue fomentar las relaciones sociales de los empleados y crear un buen ambiente de trabajo; por ejemplo, si un hospital cuenta con un buen líder el cual promueve ciertas actividades que desarrollen la empatía en los emplea- 
dos, esto puede ayudar a que los empleados tengan un mejor ambiente laboral, que permiten la confianza entre ellos.

El cuarto grupo entendía la inteligencia emocional como la capacidad para gestionar bien las propias emociones, de este modo, el líder resonante debía sintonizar con las emociones de las personas que le rodeaban. Su capacidad como líder dependía de su propia capacidad para controlar las emociones debiendo crear un entorno emocional positivo para que las relaciones de la empresa prosperasen. Además, estimaban que el líder resonante era un líder visionario a la vez que democrático, capaz de prever los cambios en la organización a la vez que sabía mantener la conexión con todos sus seguidores. Este grupo de discusión asimismo consideraba que desde el punto de vista de la inteligencia social era indispensable que el líder resonante desplegara una óptima inteligencia emocional, pues sin la inteligencia emocional no se podía conseguir la inteligencia social. Si cabe, debía poseer una sensibilidad social sin que las consecuencias negativas de la misma le afectasen.

Respecto a las desventajas del liderazgo resonante, el primer grupo consideraba que el líder estaba bajo presión ya que siempre era observado; además, en ocasiones, el involucrarse tanto en los problemas de los subordinados podría ocasionarle un conflicto personal ya que se podía sentir parte del problema cuando no lo era. Por otro lado, sino actuaba de manera correcta entonces podría convertirse en un líder disonante. Por último, todas las ventajas se podrían convertir en desventajas si el líder y el entorno no eran favorables.

El segundo grupo consideraba que, por muy fuerte que fuera el líder psíquica y físicamente, ningún directivo está libre de volverse disonante, es decir, de generar emociones adversas en el personal. Los líderes disonantes son presa del llamado "síndrome del sacrificio" que consiste en entregar más de lo que se puede. Cuando esto sucede entran en una espiral de estrés de la que es muy difícil salir. Afortunadamente, siempre es posible redimirse del estrés y volver a ser un líder resonante mediante un proceso de renovación personal. Asimismo, este grupo no encontraba muchas desventajas en este tipo de liderazgo pues lo consideraba muy beneficioso para cualquier empresa. En cualquier caso puede que, en ocasiones, el líder no pudiera ser optimista en todo momento, ni estar dedicado expresamente a la motivación de los empleados, además esto pudiera causar cierto estrés por el agotamiento de su energía, a no ser que el líder fuera optimista por naturaleza.

El tercer grupo consideraba que si el líder estaba mal emocionalmente podría provocar un "efecto dominó", es decir, podría transmitir este estado de ánimo al resto del equipo, por ejemplo, si el líder había tenido una discusión con su pareja su malestar podría provocar este mismo sentimiento en el resto del grupo.

El cuarto grupo estimaba como desventaja que si el líder resonante sufría mucho estrés, y si llegado a un punto no era capaz de gestionar esa situación, se podría llegar a encontrar en una situación de crisis. 


\section{Conclusiones}

En general en todos los grupos de discusión la percepción de este tipo de liderazgo ha sido positiva por los beneficios que procura creando un clima laboral más humano y una mayor rentabilidad en la empresa, pero también señalan unánimemente que, desplegando este tipo de liderazgo, se tiene además cierta presión, debido a que se debe mantener esa vibración emocional positiva y requiere por parte del directivo gran energía o una naturaleza optimista de por sí.

En cuanto a las características del líder resonante la muestra considera que debe desplegar entusiasmo, optimismo, empatía, competencia verbal, inteligencia emocional entendida como capacidad para gestionar bien las propias emociones donde el poder y el liderazgo se ven reforzados; además, desarrollará en concreto una óptima inteligencia emocional, pues sin inteligencia emocional no se puede conseguir inteligencia social; además si cabe poseer una sensibilidad social sin que las consecuencias negativas de la misma le afectasen.

Acerca de las acciones del líder resonante, se pueden observar cuatro tipos de acción. Primero, transmitir y saber suscitar emociones en los empleados como ayudar en el desempeño, motivar, crear confianza y armonía social, cumplir los objetivos de la empresa, generar emociones positivas (conciencia, confianza y compasión) y saber despertar sentimientos positivos de los subordinados. Segundo, saberse comunicar con los demás: ser asertivo, saber escuchar y prestar atención, sintonizar con las emociones de las personas que le rodeaban, ser atinado para analizar el lenguaje corporal de los demás, ser bueno psicoanalizando y leer entre líneas cuando los demás hablan, gestionar bien el contacto físico e interpretar correctamente las situaciones sociales que hubiere alrededor. Tercero, determinar la norma emocional del grupo: desde crear un entorno emocional positivo y proporcionar pautas para interpretar y reaccionar emocionalmente ante determinadas situaciones hasta incentivar al equipo para que aporten ideas, iniciativas y propuestas para salir de una situación difícil. Cuarto, labor previsora sobre el clima laboral: el líder resonante es visionario a la vez que democrático, pues es capaz de prever los cambios en la organización.

En cuanto a los efectos provechosos en el empleado por este tipo de liderazgo, la muestra considera que los subordinados se sienten considerados con más dignidad y con más calidad de vida, y por lo tanto, se aumenta la motivación para rendir. Por otra parte, las relaciones personales, la confianza, la armonía, la implicación, la responsabilidad y la autonomía en el trabajo mejoran.

Los efectos que prevén para la empresa como consecuencia de este tipo de liderazgo son muy beneficiosos. Entre ellos destacan: el fomento de las relaciones sociales de los empleados, una mejora en el clima laboral y de trabajo, aumento de la eficiencia y eficacia de las personas y los equipos, que los procesos de cambio y de mejora continua se agilizan, así como se mejoran las relaciones con los clientes y, como consecuencia, finalmente mejora la rentabilidad de la empresa. 
Respecto a las desventajas que encontró la muestra acerca del liderazgo resonante eran menos numerosas. Entre las que nombraron se encontraba que el líder normalmente estaba bajo presión ya que siempre era observado y puede que en ocasiones el líder no pudiera ser optimista en todo momento, ni estar dedicado expresamente a la motivación de los empleados; además, en ocasiones al involucrarse tanto en los problemas de los subordinados podría ocasionarle un conflicto personal ya que se podía sentir parte del problema cuando no lo era. Por otro lado, si no actuaba de manera correcta entonces podría convertirse en un líder disonante, pues, por muy fuerte que sea el líder psíquica y físicamente, ningún directivo está libre de volverse disonante, es decir, de generar emociones adversas en el personal provocando un "efecto dominó", es decir, transmitiendo este estado de ánimo al resto del equipo. Los líderes disonantes son presa del llamado "síndrome del sacrificio" que consiste en entregar más de lo que se puede entrando en una espiral de estrés de la que es muy difícil salir y si llegado a un punto no era capaz de gestionar esa situación, se podría llegar a encontrar en una situación de crisis. Afortunadamente, siempre es posible redimirse del estrés y volver a ser un líder resonante mediante un proceso de renovación personal. Por último, todas las ventajas se podrían convertir en desventajas si el líder y el entorno no eran favorables.

\section{Bibliografía}

BALDWIN, T. T., BOMMER, W. H., \& RUBIN, R. S. (2013). Managing organizational behavior: What great managers know and do. New York, NY: McGrawHill Irwin.

BATEMAN, T. S., \& SNELL, S. A. (2013). Management: Leading \& collaborating in a competitive world. New York: McGraw-Hill Irwin.

BOVÉE, C. L., \& THILL, J. V. (2013). Business in action (6th ed.). Boston: Upper Saddle River, NJ: Pearson.

BOYATZIS, R. E., \& MCKEE, A. (2005). Resonant leadership: Renewing yourself and connecting with others through mindfulness, hope, and compassion. Boston: Harvard Business School Press.

CARUSO, David R. y SALOVEY, Peter (2012) El directivo emocionalmente inteligente: la inteligencia emocional de la empresa. Madrid: editorial Algaba.

DENHARDT, R. B., DENHARDT, J. V., \& ARISTIGUETA, M. P. (2013). Managing human behavior in public and nonprofit organizations (3rd ed.). Los Angeles u.a.: Sage Publications.

GOETSCH, D. L., \& DAVIS, S. B. (2013). Quality management for organizational excellence: Introduction to total quality. Boston: Pearson.

GOLEMAN, D. (1995). Inteligencia emocional. Buenos Aires: Vergara.

GOLEMAN, D., BOYATZIS, R. E., \& MCKEE, A. (2004; 2002). Primal leadership: Learning to lead with emotional intelligence. Boston, Mass.: Harvard Business School Press. 
BOYATKIS, Richard y MCKEE, Annie (2002): El líder resonante crea más. Buenos Aires: Plaza Janés.

GOLEMAN, D. (2005). Inteligencia emocional en la empresa. México:Vergara.

GOLEMAN, D. (2006). Inteligencia social. New York: Bantam books.

GOLEMAN, D. (2013). Liderazgo. El poder de la inteligencia emocional. B. Ediciones. Barcelona.

HUANG, C., HSU, P., \& CHIAU, W. (2011). Perceptions of the impact of chief executive leadership style on organizational performance through successful enterprise resource planning. Social Behavior \& Personality: An International Journal, 39(7), 865-878. doi:10.2224/sbp.2011.39.7.865 [14-10-2013]

JONES, G. R., \& GEORGE, J. M. (2013). Essentials of contemporary management (5th ed.). New York, NY: McGraw-Hill Irwin.

MCKEE, A., BOYATZIS, R. E., \& JOHNSTON, F. (2008). Becoming a resonant leader: Develop your emotional intelligence, renew your relationships, sustain your effectiveness. Boston, Mass.: Harvard Business School Pub.

MULLEN, J., KELLOWAY, E. K., \& TEED, M. (2011). Inconsistent style of leadership as a predictor of safety behavior. Work \& Stress, 25(1), 41-54. DOI:10.1080 /02678373.2011.569200. [14-10-2013]

NORTHOUSE, P. G. (2009). Introduction to leadership: Concepts and practice. Los Ángeles: Sage.

PEÑA-ACUÑA, B, PARRA-MEROÑO M.C. y BELTRÁN-BUENO M.A. (2013). El liderazgo resonante en el mundo empresarial. En DURÁN MEDINA, J.F. Los investigadores hoy. Criterios de trabajo y criterios de investigación. Madrid: Vision Libros (en prensa)

RAYNER, S., \& COOLS, E. (2011). Style differences in cognition, learning, and management: Theory, research, and practice. New York: Routledge.

ROBBINS, S. P., \& JUDGE, T. A. (2013). Organizational behavior (15, global ed.). Boston u.a.: Pearson.

ROWE, W. G., \& GUERRERO, L. (2013). Cases in leadership (3rd ed.). Los Ángeles u.a.: Sage Publ.

\section{Los autores}

Beatriz Peña Acuña tiene una formación interdisciplinar de tres carreras y es Premio extraordinario de Doctorado. Como profesor contratado doctor acreditado imparte actualmente la asignatura de "Dirección de Comunicación y habilidades directivas" en el Grado de Administración y Dirección de Empresas y también otros cursos de Máster y Posgrado. Tiene varias publicaciones sobre Comunicación tanto libros, capítulos de libro como artículos en revistas de impacto y Journals. Es investigador principal del grupo de investigación internacional "Desarrollo personal" en UCAM. Ha visitado varias universidades internacionales, entre otras: New York University (EE.UU), UEA, Queen Mary College, Universidad de Londres (Inglate- 
rra), etc. Investiga acerca de la Empresa en cuanto a la Comunicación, el Desarrollo personal y la Innovación Educativa.

María Concepción Parra Meroño es profesora de Marketing, Dirección Comercial e Investigación de Mercados en la Universidad Católica San Antonio de Murcia. Doctora en Administración y Dirección de Empresas. Sus líneas de investigación se centran en el área de Comercialización e Investigación de Mercados, con especial énfasis en el estudio del comportamiento del consumidor y los medios de comunicación. Ha publicado artículos en revistas de impacto como Estudios Sobre el Mensaje Periodístico, Cuadernos de Administración y Dirección de Empresas o Personal and Ubiquitous Computing y es autora de varios libros y capítulos sobre Marketing y Dirección Comercial.

Miguel Ángel Beltrán Bueno es licenciado en Ciencias Económicas y Empresariales. Profesor de la Universidad Católica San Antonio de Murcia donde imparte las asignaturas de Marketing, Dirección Comercial, y Comportamiento del Consumidor. Cuenta con una amplia experiencia profesional como economista en el sector privado. Ha publicado artículos en revistas de impacto y es autor de varios libros y capítulos sobre Marketing y Dirección Comercial. 